Research Paper

\title{
Differential Targeting of Stem Cells and Differentiated Glioblastomas by NK Cells
}

\author{
Han-Ching Tseng1\#, Akihito Inagaki ${ }^{4 \#}$, Vickie T. Bui ${ }^{1}$, Nicholas Cacalano ${ }^{2,3}$, Noriyuki Kasahara ${ }^{4}$, Yan-gao \\ Man $^{5}$ and Anahid Jewett ${ }^{1,2}$ \\ 1. Division of Oral Biology and Oral Medicine, The Jane and Jerry Weintraub Center for Reconstructive Biotechnology \\ 2. The Jonsson Comprehensive Cancer Center \\ 3. Department of Radiation Oncology, Division of Molecular and Cellular Oncology UCLA School of Medicine \\ 4. University of Miami, FL, USA \\ 5. Research Laboratory and International Collaboration, Bon Secours Cancer Institute, Bon Secours Health System, Richmond, VA \\ \# These authors contributed equally to the paper. \\ $\square$ Corresponding author: Address: 10833 Le Conte Ave, UCLA School of Dentistry, Los Angeles, CA 90095. Telephone: (310) 206-3970; Fax: \\ (310) 794-7109; E-mail: ajewett@ucla.edu
}

() 2015 Ivyspring International Publisher. Reproduction is permitted for personal, noncommercial use, provided that the article is in whole, unmodified, and properly cited. See http://ivyspring.com/terms for terms and conditions.

Received: 2015.01.08; Accepted: 2015.02.21; Published: 2015.07.16

\begin{abstract}
We have recently shown that Natural Killer (NK) cells control survival and differentiation of Cancer Stem-like Cells (CSCs) through two distinct phenotypes of cytotoxic and anergic NK cells, respectively. In this report, brain CSCs and their serum and NK cell differentiated counterparts were studied. Serum-differentiated brain CSCs were significantly less susceptible to NK cells and CTL direct cytotoxicity as well as NK cell mediated Antibody Dependent Cellular Cytotoxicity (ADCC), whereas their CSCs were highly susceptible. The levels of CD44 and EGFR were higher in brain tumor CSCs when compared to the serum-differentiated tumors. No differences could be observed for the expression of MHC class I between brain tumor stem cells and their serum-differentiated counterparts. Moreover, supernatants from the combination of IL-2 and anti-CD $16 \mathrm{mAb}$ treated NK cells (anergized NK cells) induced resistance of brain tumor CSCs to NK cell mediated cytotoxicity. Unlike serum-differentiated CSCs, NK supernatant induced differentiation and resistance to cytotoxicity in brain CSCs correlated with the increased expression of CD54 and MHC class I. The addition of anti-MHC class I antibody moderately inhibited NK mediated cytotoxicity against untreated or serum-differentiated CSCs, whereas it increased cytotoxicity against NK supernatant differentiated tumors. Therefore, two distinct mechanisms govern serum and NK supernatant mediated differentiation of brain tumors.
\end{abstract}

Key words: IFN- $\gamma$, NK, GBMs, brain stem cells

\section{Introduction}

Glioblastomas (GBMs) are among the tumors with poor prognosis. The current chemo- and radio-therapeutic strategies against GBMs have had limited success in either controlling the disease or establishing long lasting regression of these tumors in the patients, therefore, there is a clear need for effective therapies to control these tumors. There is also evidence that the differentiation status of tumor cells might affect their therapeutic outcome [1]. GBMs contain self-renewing, stem-like subpopulation with the ability to sustain tumor growth. These cells, also known as Cancer Stem-like Cells (CSC), share certain phenotypic characteristics with untransformed stem cells and are resistant to chemo- and radio-therapy due to increased expression of multi-drug resistance and DNA mismatch repair genes. In recent years, T cell based immune-therapies have gained some popularity and were shown to have some success in 
treatment of certain patients, however, because of a lower MHC class I expression on GBMs the effectiveness of CTL based therapies may be limited. On the other hand, NK cells should be able to target such tumors since they are known to kill tumors with no or decreased levels of MHC class I expression. Indeed, it is shown that NK cells are recruited to the brain and CNS during pathological diseases such as Multiple Sclerosis and during non-pathological human brain and have a significant role in immune regulation [2]. In GBMs NK cells are found in the tumor microenvironment and are shown to be suppressed by the tumor and the effectors of the immune system [2]. In addition, targeting GBMs with the NK cells increases the survival of the animals [3]. Therefore, it is clear from the previous studies that NK cells based immunotherapies may have beneficial effect in patients.

Immunosuppression and tumor escape from immune recognition are thought to be major factors responsible for the establishment and progression of cancer, however, neither underlying physiological relevance nor the exact mechanisms by which immunosuppression occurs are well understood. It is shown that freshly isolated tumor infiltrating NK cells are not cytotoxic to autologous tumors. Moreover, NK cells obtained from the peripheral blood of patients with cancer have significantly reduced cytotoxic activity [4-7]. In addition, NK cell cytotoxicity is suppressed after their interaction with stem cells [8-10]. In contrast, interaction of NK cells with the resistant tumors did not result in suppression of NK cell cytotoxicity $[11,12]$.

Increased NK cell cytotoxicity and augmented secretion of IFN- $\gamma$ were observed against Oral Squamous Cancer Stem Cells (OSCSCs) when compared to differentiated Oral Squamous Carcinoma Cells (OSCCs) [13]. More importantly, OSCSCs expressed CD44 high CD326 high CD26 high CD166 low CD338 $^{+}$oral stem cell markers $[1,13]$. We have also demonstrated that NK cells were able to lyse hMSCs, hDPSCs, hESCs and hiPSCs significantly more than their differentiated counterparts [13]. In addition, de-differentiation by blocking $\mathrm{NF \kappa B}$ in oral tumors was able to increase NK cell mediated cytotoxicity [13-15] and elevated CD44 surface receptor expression (data not shown).

We have previously shown that K562, an NK sensitive tumor, causes loss of NK cell cytotoxicity while increasing IFN- $\gamma$ secretion by the NK cells, a term which we coined as split anergy [11, 15-20]. On the other hand NK resistant tumors, such as RAJI cells, do not induce split anergy in NK cells $[11,18]$. Significant down-modulation of CD16 receptor and decreased NK cell cytotoxic function were also seen in patients with cancer including those of the oral and ovarian cancer patients [21,22].

In this paper we studied the function of NK cells against three GBM CSCs, their serum-differentiated and NK supernatant differentiated counterparts. Overall, our data indicates that there is heterogeneity within the subpopulations of GBM CSCs as seen by the levels of CSC surface marker expression and susceptibility to NK cell mediated cytotoxicity. In addition, NK cells are highly effective in eliminating CSCs in GBMs. However, differentiation and resistance of GBM tumors by the factors present in serum and those secreted by anergized NK cells may limit lysis of tumors by competent NK cells. Such decrease in lysis of differentiated GBM tumors by the NK cells compounded by the known inactivation of NK cell cytotoxic function by the effectors of the tumor microenvironment may significantly curtail effectiveness of NK cells in controlling the progression of GBM tumors.

\section{Materials and Methods}

\section{Cell Lines, Reagents, and Antibodies}

RPMI 1640 supplemented with 10\% FBS was used for the cultures of human NK cells. Stem-like GBMs were isolated from freshly resected human tumor tissues as described previously [23], and they were seeded at $1 \times 10^{5}$ cells $/ \mathrm{mL}$ into culture flasks pre-coated with laminin $(1 \mathrm{mg} / \mathrm{mL}$; BD Biosciences, CA) and Poly-L-Ornithine $(15 \mathrm{ug} / \mathrm{mL}$; Sigma Aldrich, MO). The cells were cultured in DMEM/F12 medium (Gemini Bio-Products, CA) supplemented with penicillin G, streptomycin sulfate, B-27 (1:50; Gemini Bio-Products, CA), recombinant human Fibroblast Growth Factor (hFGF-2, 20ng/mL, R\&D Systems, $\mathrm{MN})$, recombinant human Epidermal Growth Factor (hEGF, 20ng/mL, R\&D Systems, MN) and Leukemia Inhibitory Factor (LIF, 1000U/mL). The medium was refreshed every 3 days. Recombinant IL-2 was obtained from NIH-BRB. The human NK purification kits were obtained from Stem Cell Technologies (Vancouver, Canada). The antibodies for CD16 and CD44 were obtained from Biolegend (San Diego, CA). Anti-MHC class I were prepared in our laboratory and 1:100 dilution was found to be the optimal concentration to use. EGFR antibody (Erbitux) was purchased from UCLA pharmacy.

\section{Transplantation of CSCs into the immunode- ficient mice}

Tumor formation was performed as described previously [23]. 45-47 days later, all mice injected with two glioblastoma CSCs X01GB, X02GB and one anaplastic oligoastrocytoma X03AOA developed malig- 
nant brain tumors. Intracranial tumors generated by all three tumor lines demonstrated high infiltration into the surrounding cerebral cortex as evidenced by an unclear demarcation between the tumors and the normal tissues.

\section{Purification of NK cells}

NK cells were purified from healthy donors using negative isolation kits from Stem Cell Technologies, Vancouver, Canada, as described previously [11]. Written informed consents approved by UCLA Institutional Review Board (IRB) were obtained from the blood donors and all the procedures were approved by the UCLA-IRB.

\section{Purification of $\mathbf{T}$ cells and the generation of CTLs}

X02GB cells were irradiated at 7,000 rads from a ${ }^{60} \mathrm{Co}$ source. PBMCs were cultured with X02GB cells for 14 days at a responder to stimulator ratio of 15:1 in RPMI-1640 containing 10\% FBS and 60 IU IL-2/mL. Daily lactic acid concentrations were used to determine if replenishment with fresh culture medium was necessary. $\mathrm{CD}^{+} \mathrm{T}$ cells were purified negatively using $\mathrm{T}$ cell isolation kit (Stem Cell Technologies, Vancouver, Canada).

\section{GBM CSCs differentiation with serum or NK cell supernatant}

To differentiate with serum, the brain CSCs were cultured in DMEM medium supplemented with $10 \%$ FBS for a period of 4 weeks. To differentiate with NK cell supernatants, NK cells were left untreated or treated with the combination of anti-CD $16 \mathrm{mAb}$ (3ug/ml) and IL-2 (1000 units/ml) for 18- 24 hours before the supernatants were removed and used in differentiation experiments. The amounts of IFN- $\gamma$ produced by activated NK cells were assessed with IFN- $\gamma$ ELISA (Biolegend, CA). Serum-differentiated XO2GB (XO2GB-S) were further selected for differentiation with the NK cell supernatants. Differentiation of XO2GB-S with NK cell supernatants was conducted with gradual daily addition of increasing amounts of NK cell supernatants. XO2GB-S required on average a total of $0.035 \mathrm{pg}$ of IFN- $\gamma$ containing supernatants from IL-2+anti-CD16mAb treated NK cells per tumor cell during a 7 day treatment, whereas XO2GB CSCs required $0.070 \mathrm{pg}$ of IFN- $\gamma$ containing supernatants from IL-2+anti-CD16mAbmAb treated NK cells per tumor cell for 7-10 days to promote differentiation and resistance to NK cell mediated cytotoxicity. Initially $1 \times 10^{6}$ tumor cells were cultured and treated with NK supernatants for differentiation. Afterwards, target cells were rinsed with 1X PBS, detached and used for experiments.

\section{Surface Staining}

Staining was performed by labeling the cells with antibodies as described previously [11, 17, 24].

\section{${ }^{51} \mathrm{Cr}$ release cytotoxicity assay}

The ${ }^{51} \mathrm{Cr}$ release assay was performed as described previously [25] and the LU $30 / 10^{6}$ was calculated by using the inverse of the number of effector cells needed to lyse $30 \%$ of target cells X100.

\section{Statistical analysis}

An unpaired, two-tailed student t-test was performed for the statistical analysis. One way ANOVA with a Bonferroni post-test was used to compare the different groups.

\section{Results}

Characterization of brain tumor stem cells and their serum-differentiated counterparts

X01GB and X02GB and X03AOA CSCs [23, 26, 27] and their serum-differentiated counterparts (XO1GB-S, XO2GB-S and XO3AOA-S) were used to determine surface expression of key receptors. The brain CSCs exhibited a rounded morphology, which upon differentiation with serum demonstrated flat fibroblast-like spindle shape cells (Fig. 1A). CSCs formed tumors in the brain of immunodeficient mice with high proliferative capacity (Fig. 1B). The levels of CD44 were the highest in X01GB and X02GB and lower in X03AOA CSCs (Fig. 1C). Serum-differentiation caused significant decreases of CD44 surface expression in all 3 CSCs; however, X03AOA exhibited the least decrease in CD44 expression after differentiation (Fig. 1C). EGF receptor expressions were significantly higher in all 3 brain CSCs when compared to their differentiated counterparts (Fig. 1C). X02GB had the highest EGF receptor expression, followed by X03AOA and the least expression was observed on X01GB. In contrast, no consistent differences could be observed for the expression of MHC class I and the levels were not changed significantly after differentiation of CSCs (data not shown). The profiles of U87 GBM cell line were similar to those of the differentiated primary GBM tumors (Fig. 1C).

\section{X02GB CSCs were lysed significantly more than X01GB and X03AOA CSCs.}

Both untreated and IL-2 treated NK cells mediated the highest lysis against $\mathrm{X} 02 \mathrm{~GB}$, followed by X01GB and the least NK cell mediated lysis was observed against X03AOA (Fig. 2A). Treatment of NK cells with anti-CD16mAb with and without IL-2 significantly diminished NK cell cytotoxicity against all three brain tumor cells. Lysis of brain CSCs by NK 
cells was significantly more when compared to U87 GBM cell line (Fig. 2A).

\section{Differentiation of brain CSCs with serum de- creased susceptibility to NK cell mediated cytotoxicity}

Differentiation of all three brain CSCs with serum resulted in a significant decrease in NK cell mediated cytotoxicity (Fig. 2B). Decrease in NK cell mediated cytotoxicity was observed in untreated as well as IL-2 and IL-2+anti-CD16mAb treated NK cells when added to serum-differentiated brain tumor cells (Fig. 2B). The highest decrease in NK cell mediated cytotoxicity could be observed in the most sensitive stem cells X02GB after differentiation. XO3AOA had the lowest decrease in IL-2 mediated cytotoxicity when differentiated (Fig. 2B).

\section{Differentiation of stem cells with serum de- creased expression of EGFR and NK cell ADCC against brain tumors}

The levels of ADCC were found to be different from direct cytotoxicity and it correlated with the levels of expression of EGFR on the surface of the brain stem cells (Figs. 1C and 2C). X02GB had the highest expression of EGFR (Fig. 1C) and therefore, NK cells mediated the highest levels of ADCC against these cells (Fig. 2C). X03AOA expressed the next highest EGFR expression therefore, unlike the direct cytotoxicity where NK cells mediated the least cytotoxicity they had the second highest ADCC when compared to the other 2 cell lines (Fig. 2C). The lowest ADCC was observed against X01GB which expressed the lowest surface expression of EGFR (Fig. 2C).

Differentiation of brain CSCs by serum decreased direct cytotoxicity as well as NK cell mediated ADCC (Fig. 2C). Differentiation of the CSCs by serum decreased the levels of EGFR expression on brain tumor cells and resulted in a decreased NK cell mediated ADCC against X02GB and X03AOA tumors (Figs. $1 \mathrm{C}$ and $2 \mathrm{C}$ ). Similar results were obtained when differentiated XO1GB were used with NK cells in the presence of EGFR antibody Cetuximab (data not shown).

B

X01GB 47 Days
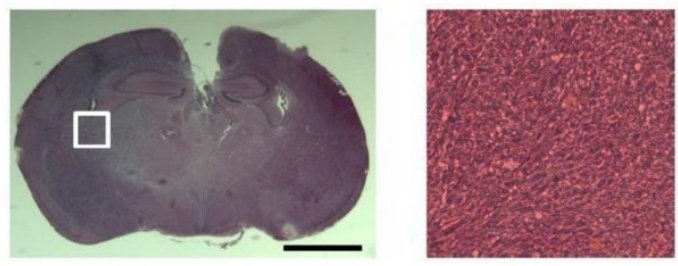

X01GB
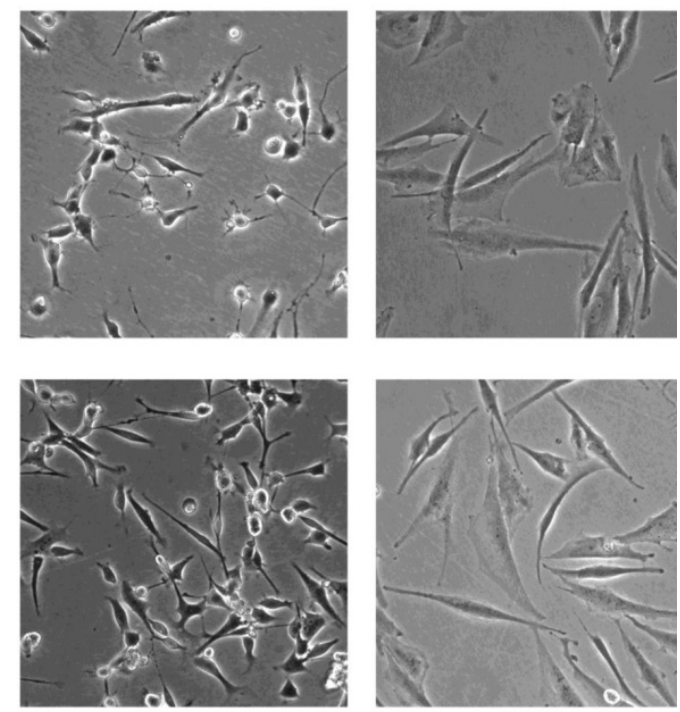

X02GB

46 Days
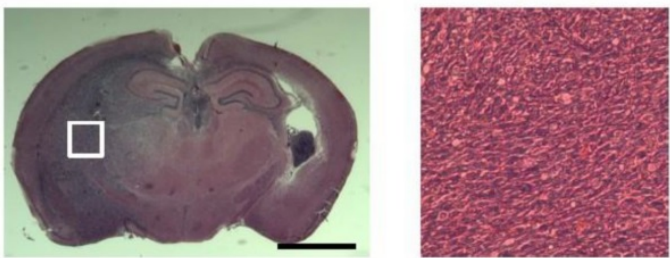

X03AOA 45 Days
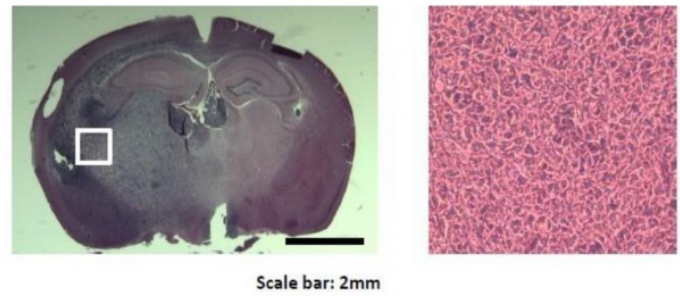

X03AOA
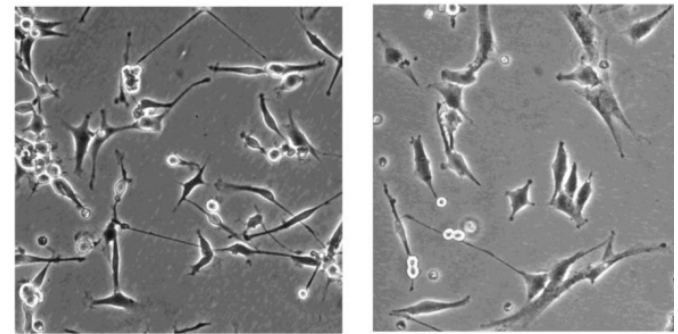
C



Isotype EGFR
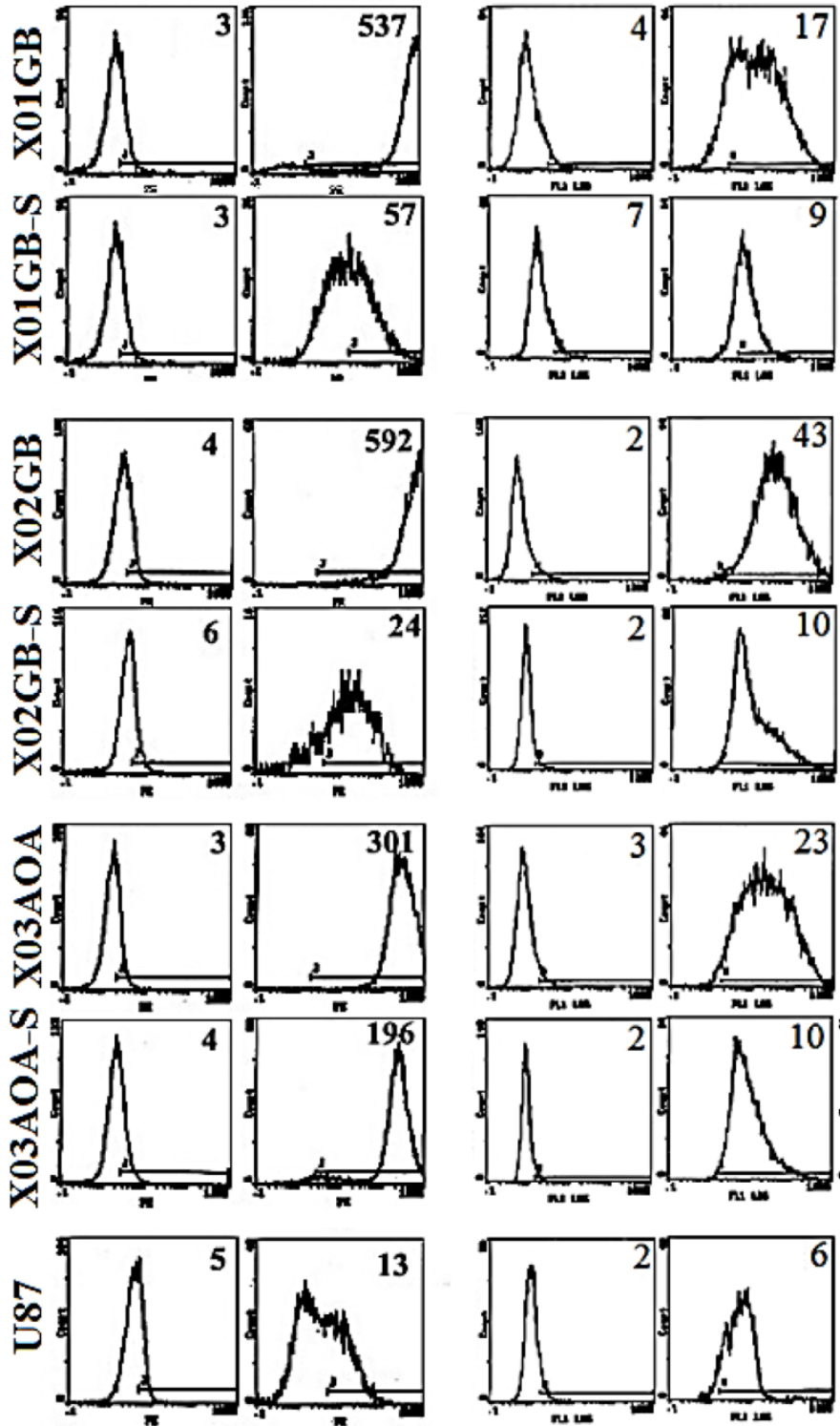

Fig. 1. X01GB, X02GB and X03AOA stem cells expressed significantly higher surface expression of CD44 and EGFR when compared to their serum-differentiated counterparts. X01GB, X02GB and X03AOA cells were cultured in either non-serum (NS) culture or differentiated in the presence of serum as described in the Materials and Methods section. As shown in the figure the brain stem cells exhibited more of a rounded morphology, which upon differentiation with serum demonstrated flat fibroblast-like spindle shape cells (A) X01GB, X02GB and X03AOA cells ( $2 \times 10^{5}$ cells) were injected orthotopically into the brain of immunodeficient nude mice as described in the Materials and Methods section and histological analysis was performed by H\&E staining. Right panel in Fig. $1 \mathrm{~B}$ demonstrated that these tumors have high proliferative capacity in vivo (B). X01GB, X02GB and X03AOA stem cells and their serum-differentiated counterparts were stained with either isotype control antibody or PE conjugated antibodies to CD44 mAb (left panel), EGF-R mAb (right panel) as described in the Materials and Methods section. The levels of CD44 and EGF receptor expression on each cell type were determined by flow cytometric analysis. The numbers on the upper right hand corner of each histogram are the mean channel fluorescent intensities. One of three representative experiments is shown in this figure. 
A
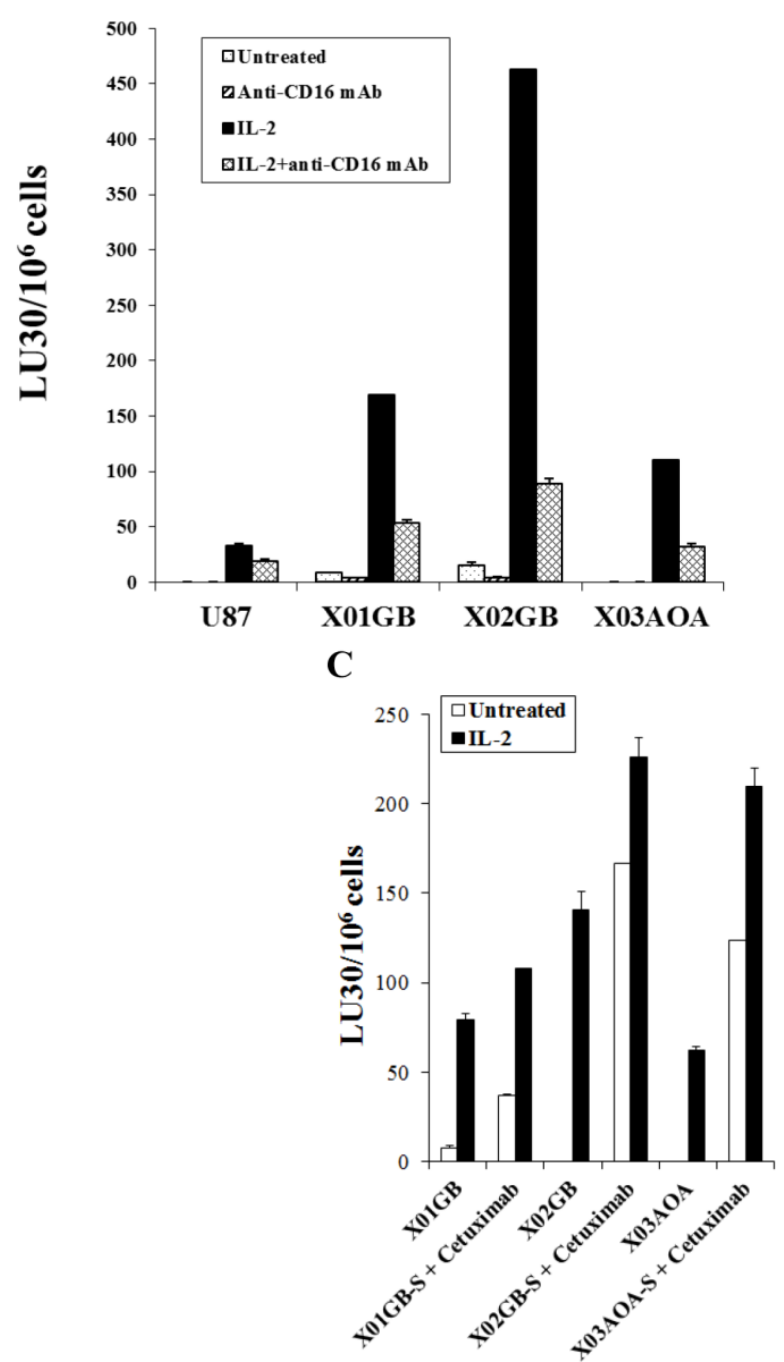

B
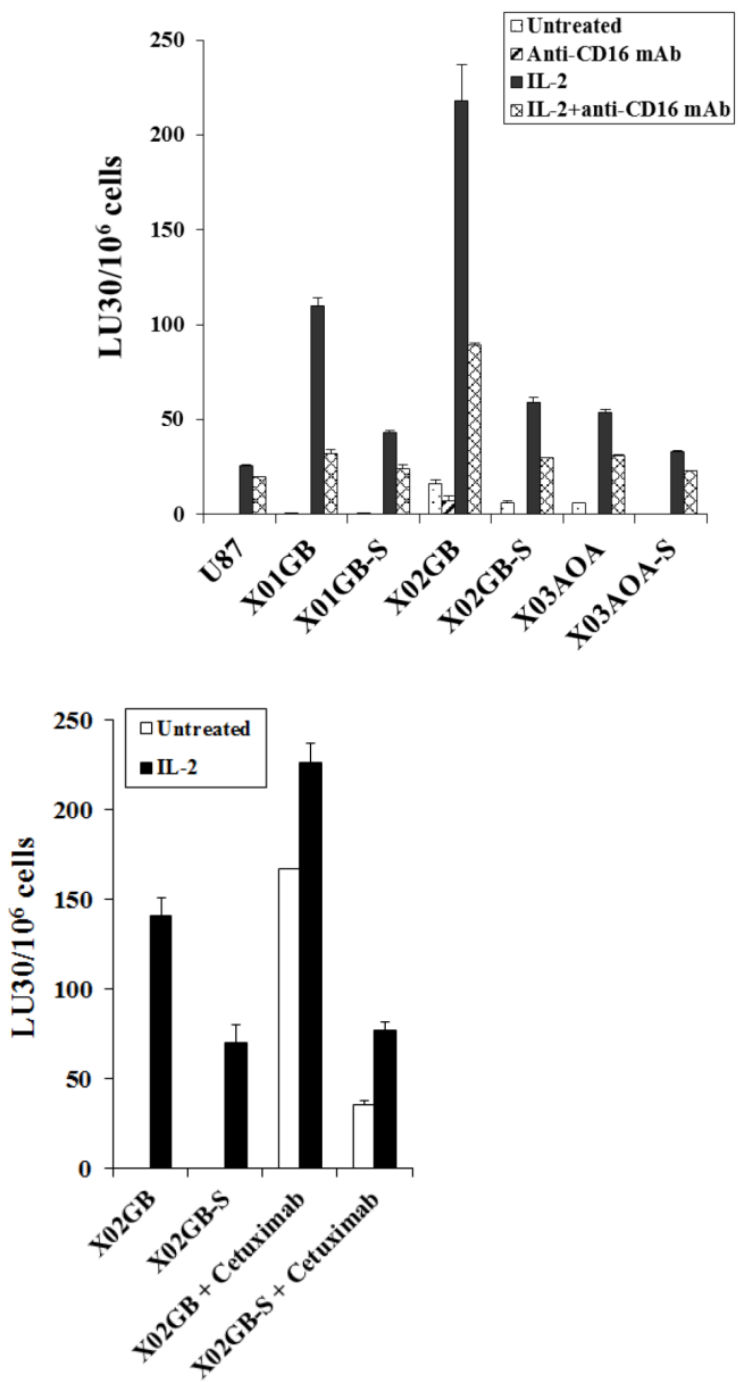

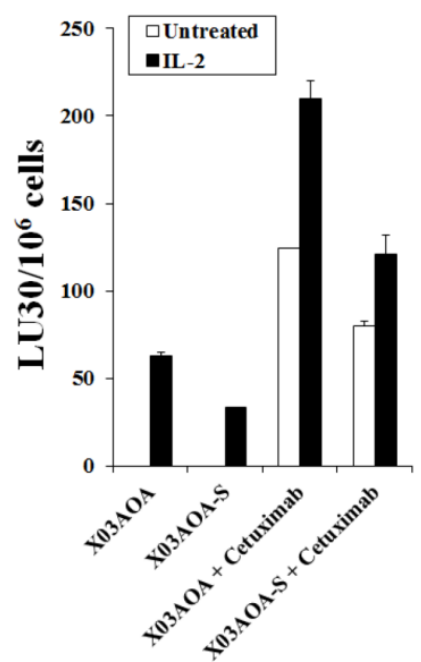

Fig. 2. X02GB were lysed significantly more than X01GB and X03AOA CSCs, and serum-differentiation decreased their susceptibility to NK cell mediated cytotoxicity and increased their resistance against NK cell mediated ADCC. Highly purified NK cells (1X106 cells/ml) were either left untreated or treated with $\mathrm{IL}-2(1000 \mathrm{u} / \mathrm{ml})$, anti-CD $16 \mathrm{mAb}(3 \mu \mathrm{g} / \mathrm{ml})$ or a combination of IL-2 $(1000 \mathrm{u} / \mathrm{ml})$ and anti-CD $16 \mathrm{mAb}(3 \mu \mathrm{g} / \mathrm{ml})$ for $12-24$ hours and used in cytotoxicity assay against X01 GB, X02GB, X03AOA stem cells and U87 GBMs (A) and along with their serum-differentiated counterparts (B). Purified NK cells were left untreated or treated with IL-2 (1000 units/ml) for $12-24$ hours and then added to ${ }^{51} \mathrm{Cr}$ labeled X01GB, X02GB and X03AOA stem cells and their serum-differentiated counterparts treated with and without Cetuximab ( $\left.10 \mu \mathrm{g} / \mathrm{ml}\right)$ (C). NK cell cytotoxicities were determined using a standard ${ }^{51} \mathrm{Cr}$ release assay and the lytic units $30 / 10^{6}$ were determined using inverse number of effectors required to lyse $30 \%$ of the tumor cells X 100 in Figs A-C. Differences between untreated or IL-2 and/or anti-CD16mAb treated NK cell killing against all 4 cell lines were significant at a p value of $<0.05$ in Figs A-C. One of four representative experiments is shown in this figure. 


\section{T cells lysed brain tumor stem cells more than their serum-differentiated counterparts}

Cytotoxic $T$ cells were generated against XO2GB and used in killing assay against both the stem cells and their differentiated counterparts. As shown in Fig. $3 \mathrm{CD}^{+} \mathrm{T}$ cells purified from PBMCs pre-stimulated with irradiated $\mathrm{XO} 2 \mathrm{~GB}$ to generate CTLs killed XO2GB stem cells, but they were unable to lyse their differentiated counterparts. The addition of anti-MHC-Class I antibody significantly blocked CTL mediated lysis of XO2GB stem cells. CTLs were unable to lyse either NK cell sensitive K562 or NK cell resistant Raji or NK cell sensitive OSCSCs (Fig. 3). The levels of CTL mediated lysis of XO2GB stem cells were significantly less than that mediated by the NK cells obtained from the same donor (Supplementary Fig. S1A). The addition of anti-MHC class I antibody did not change the resistance of XO2GB-S cells against CTLs. In contrast to CTLs, NK cells purified from the same donor significantly lysed OSCSCs and K562s, but not Raji cells (Figs. S1A and S1B) and the addition of anti-MHC class I antibody inhibited NK cell mediated lysis of OSCSCs similar to that seen when anti-MHC class I antibody was added to the cultures of CTLs with XO2GB stem cells (Supplementary Fig. S1A).

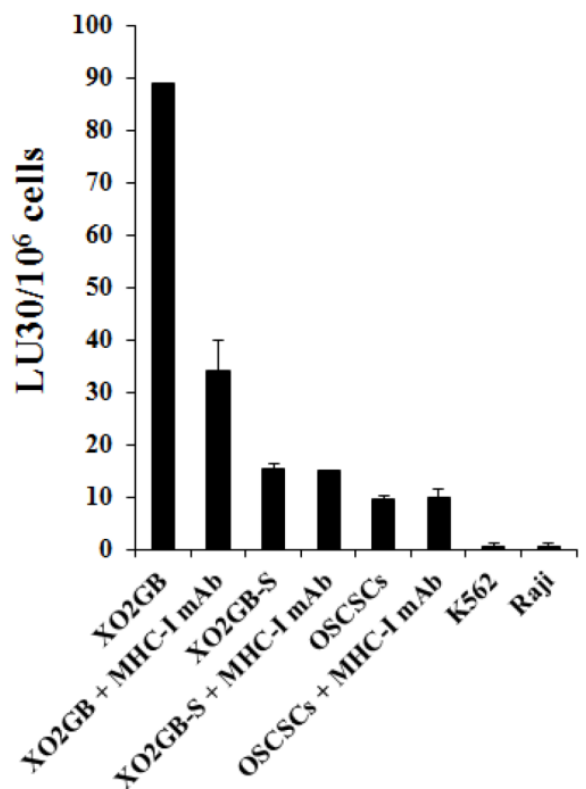

Fig. 3. CTLs mediated cytotoxicity against stem-like GBMs but not their serum-differentiated counterparts. $X 02 \mathrm{~GB}$ were irradiated at 7,000 rads from a ${ }^{60} \mathrm{Co}$ source. PBMCs were cultured with $\mathrm{X02GB}$ for 14 days at a responder to stimulator ratio of $15: 1$ in RPMI-1640 containing 10\% FBS and $60 \mathrm{IU} \mathrm{IL-2} / \mathrm{mL}$. Daily lactic acid concentrations were used to determine if replenishment with fresh culture medium was necessary. $C D 8^{+} \mathrm{T}$ cells were purified negatively using $\mathrm{T}$ cell isolation kit and cultured with stem-like ${ }^{51} \mathrm{Cr}$ labeled XO2GB and XO2GB-S at different E:T ratios and cytotoxicity were determined in a 4 hour ${ }^{51} \mathrm{Cr}$ release assay. Anti-MHC class 1 antibody was added at a 1:100 dilution. NK sensitive OSCSCs and K562 and NK resistant Raji cells were used as controls. Supernatants were then harvested and radioactivity counted using a gamma counter. Lytic units 30/106 cells were determined using inverse number of effector cells required to lyse $30 \%$ of the target cells $X$ 100.

\section{Supernatants from IL-2+anti-CD $16 \mathrm{mAb}$ treated NK cells induced resistance of XO2GB-S to NK cell mediated cytotoxicity}

We next determined differences between non-serum treated XO2GB, XO2GB-S, and XO2GB-S differentiated by supernatants from the IL-2+anti-CD16mAb treated NK cells in regards to resistance to NK cell mediated cytotoxicity. Treatment of XO2GB-S with IL-2+anti-CD16mAb treated NK cell supernatants decreased NK cell mediated cytotoxicity significantly by freshly isolated untreated, IL-2 treated or IL-2+anti-CD16mAb treated NK cells $(\mathrm{P}<0.05)$ (Fig. 4A). Resistance of XO2GB-S to NK cell mediated cytotoxicity could also be observed after their treatment with supernatants from IL-2 treated NK cells, however, the levels of resistance were significantly less when compared to those induced by IL-2+anti-CD16mAb treated NK cell supernatants (data not shown). The following profile emerged from these studies. XO2GB in the absence of serum were the most sensitive to NK cell mediated cytotoxicity, followed by XO2GB-S and the most resistance could be observed when supernatants from IL-2+anti-CD16mAb treated NK cells were used to differentiate XO2GB-S (Fig. 4A).

When non-serum treated $\mathrm{XO} 2 \mathrm{GBs}$ were differentiated with supernatants from IL-2+anti-CD16mAb treated NK cells $68 \%$ inhibition in the cytotoxicity could be observed when untreated NK cells were used to assess cytotoxicity. However, only 36\% decrease could be seen with IL-2 treated NK cells, whereas with XO2GB-S 75\% and 74\% inhibition with untreated and IL-2 treated NK cells could be seen, respectively. These experiments indicated that supernatants from IL-2+anti-CD16mAb treated NK cells were able to induce resistance to XO2GB-S more efficiently than non-serum treated XO2GBs.

\section{Induction of NK resistance in XO2GB-S by supernatants from IL-2+anti-CD $16 \mathrm{mAb}$ treated NK cells correlated with the increased expression of CD54 and MHC class I}

We then assessed correlation between resistance to NK cells induced by the supernatants from IL-2+anti-CD16mAb treated NK cells in XO2GB-S with key cell surface receptor expression. Among many surface receptors tested CD54 and MHC class I expression were found to correlate significantly with the differentiation and resistance of NK sup-differentiated XO2GB-S (Fig. 4B). As shown in Fig. 4B, the levels of CD54 and MHC class I increased substantially on XO2GB-S in the presence of 
IL-2+anti-CD16mAb treated NK cell supernatants. Supernatants from untreated NK cells did not have significant effect on surface expression of XO2GB-S
(Fig. 4B). No significant differences could be seen for the expression of CD54 and MHC class I expression between XO2GB CSCs and XO2GB-S cells (Fig. 4B).

A

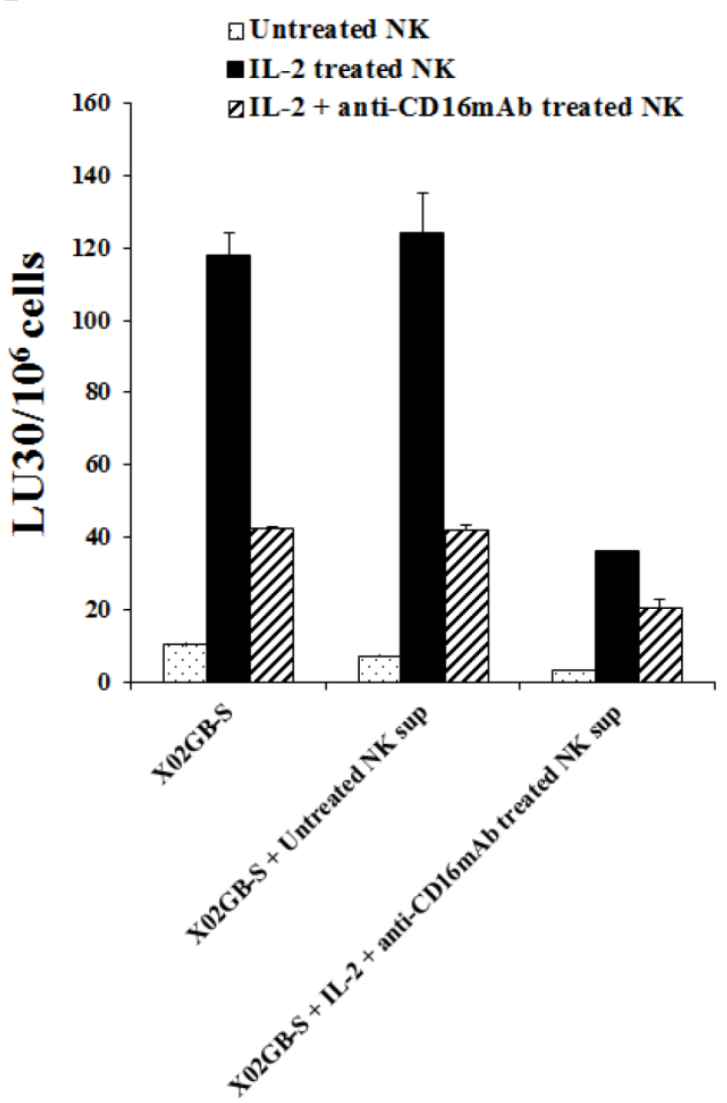

B

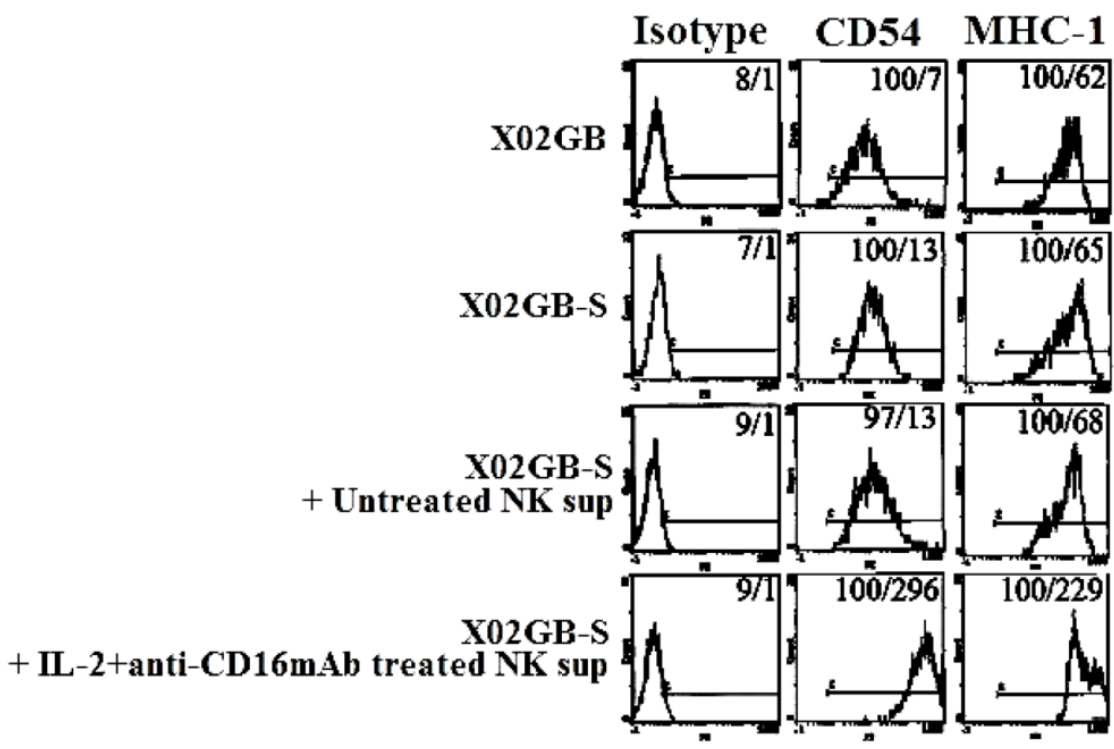

Fig. 4. Increased resistance to NK cell mediated cytotoxicity and increased expression of CD54 and MHC class I on X02GB-S differentiated with supernatants from IL-2+anti-CD 16mAb treated NK cells. Highly purified NK cells were left untreated or treated with the combination of IL-2 (1000 units/ml) and anti-CD $16 \mathrm{mAb}(3 \mu \mathrm{g} / \mathrm{ml})$ for 24 hours, after which the supernatants were removed and used for the treatment of X02GB-S cells for 7 days. X02GB-S in the absence of NK supernatants was used as controls. Similar amounts of supernatants from untreated NK cells and those cultured with IL-2+anti-CD16mAb treated NK cells were used to treat X02GB-S to induce differentiation. Afterwards, the cells were rinsed with 1 X PBS, detached from the tissue culture plate and used in a standard ${ }^{51} \mathrm{Cr}$ release assay against freshly isolated untreated NK, IL-2 (1000 units/ml) and the combination of IL-2 (1000 units/ml) and anti-CD16mAb $(3 \mu \mathrm{g} / \mathrm{ml})$ treated NK cells $(\mathrm{A})$. X02GB-S was differentiated with NK supernatants as described in figure 4A and the surface expression of CD54 and MHC class I on untreated X02GB, untreated X02GB-S and NK supernatant treated X02GB-S were assessed after 7 days of differentiation using PE conjugated antibodies followed by flow cytometric analysis. Isotype control antibodies were used as controls. The numbers on the right hand corner are the percentages and the mean channel fluorescence intensities in each histogram (B). 
A

B


C



Fig. 5. Antibody to MHC-class I inhibited NK cell mediated cytotoxicity moderately against stem-like XO2GB and XO2GB-S whereas it increased substantially against IL-2+anti-CD16mAb NK supernatant differentiated XO2GB-S. NK cells $\left(1 \times 10^{6} / \mathrm{ml}\right)$ were treated with IL-2 (1000 units $\left./ \mathrm{ml}\right) 12-24$ hours before they were cultured with ${ }^{51} \mathrm{Cr}$ labeled XO2GB (A), XO2GB-S (B) or NK supernatant differentiated X02GB-S cells $(C)$ at different $E: T$ ratios in the presence and absence of anti-MHC-Class I mAb (1:100 dilution). X02GB-S differentiation by NK supernatant was performed as described in figured $4 A$. Percent cytotoxicity was obtained at different effector to target ratio, and the lytic units $30 / 10^{6}$ cells were determined using inverse number of NK cells required to lyse $30 \%$ of the tumor cells $\times 100$.

MHC class I antibody augmented NK cell cytotoxicity against IL-2+anti-CD 16 mAb treated NK supernatant differentiated XO2GB-S whereas it inhibited cytotoxicity against XO2GB CSCs

As shown in Fig. 5, the addition of anti-MHC class I $\mathrm{mAb}$ to the cultures of NK cells with stem like XO2GB (Fig 5A) or XO2GB-S (Fig. 5B) decreased NK cell cytotoxicity by $36.5 \%$ and $16.4 \%$, respectively, whereas it increased NK cell cytotoxicity (56.9\%) against XO2GB-S differentiated with the supernatants from IL-2+anti-CD16mAb treated NK cells (Fig. 5C). Increase in $\mathrm{NK}$ cell cytotoxicity in the presence of MHC class I antibody in IL-2+anti-CD16mAb supernatant differentiated XO2GB-S correlated with the increased expression of MHC class I on XO2GB-S (Fig. $4 \mathrm{~B})$. Both XO2GB and XO2GB-S in the absence of supernatants from IL-2+anti-CD16mAb treated NK cells exhibited no or lower expression of MHC class I (Fig. 4B). Similarly, anti-MHC class I mAb decreased NK cell mediated cytotoxicity when added to the cultures of NK cells with OSCSCs, whereas it increased when added to IL-2+anti-CD16mAb treated NK supernatant differentiated OSCSCs (Supplementary Fig. S1A and [1]). The addition of antibodies to MHC class I increased NK cell cytotoxicity significantly when added to well- differentiated OSCCs isolated from oral cancer patient which exhibited higher surface expression of MHC class I [1].

\section{Discussion}

In this report we present evidence that NK cells target brain cancer stem cells significantly more than their differentiated counterparts. The phenotypic characteristics and the nature of stemness of the three brain tumor stem cells used in our study has been established and reported previously [26]. Our results demonstrated that NK cells were able to lyse X02GB tumors significantly more than either X01GB or X03AOA brain tumor stem cells. The lysis of brain tumor stem cells were closely correlated with the expression of CD44 levels on the cells. Serum-differentiation of brain stem cells decreased CD44 expression on all the lines, however, more dramatic decreases could be observed for X02GB and X01GB and less for X03AOA cells.

Dissociation between direct cytotoxicity and ADCC was found when NK cell cytotoxicity was measured against X01GB and X03AOA brain stem cells. Whereas NK cells mediated lower levels of direct cytotoxicity against $\mathrm{X} 03 \mathrm{AOA}$ when compared to X01GB, the levels of NK cell ADCC was higher against X03AOA brain stem cells as compared to X01GB in the presence of Cetuximab, and it correlated with the higher expression of EGFR on X03AOA. Interestingly, the levels of EGFRvIII gene expression was higher in X01GB and X03AOA stem cells and no expression could be seen in X02GB [26]. These results may indicate different levels of stemness in the three brain stem cells, and the ability of NK cells to be the predictor of the levels of stemness in the tumors.

Both NK and CTLs were able to lyse XO2GB stem cells significantly more than their differentiated counterpart, albeit the levels of NK cell cytotoxicity were much higher than those obtained by CTLs. Indeed, peptide specific targeting of GBM stem-like 
tumors but not their serum-differentiated counterparts by specific CTLs was shown previously [28]. As expected, the addition of anti-MHC-Class I antibody inhibited CTL mediated lysis of XO2GB, and moreover, CTLs were not able to lyse NK cell sensitive K562 or OSCSCs.

The magnitude of NK cell cytotoxicity in the presence of MHC class I mAb correlated with the levels of MHC class I surface expression on tumors. Inhibition of NK cell cytotoxic function by anti-MHC class I antibody was seen in the presence of GBM and OSCSC stem-like cells which expressed very low levels of MHC class I, whereas increased cytotoxicity was seen when the cells were differentiated which expressed higher levels of MHC class I. Stem-like tumors which were NK cells supernatant differentiated or were isolated from well differentiated tumors exhibited increased NK cell mediated cytotoxicity in the presence of anti-MHC class I antibody correlating with the increased expression of surface MHC class I. Indeed, we have previously demonstrated that anti-MHC class I antibody when added in the absence of CD16 receptor triggering on NK cells had no inhibitory activity on cytotoxic function of NK cells, however, it mediated significant inhibition of NK cell cytotoxicity when added in the presence of CD16 receptor triggering [19]. Based on these observations we speculated that since XO2GB and OSCSCs mediate significant receptor mediated activation of NK cells, the addition of anti-MHC class I antibody may serve to inhibit NK cell mediated cytotoxicity as seen in Figs. 5A and S1A. However, when stem cells are differentiated, since NK cells are not activated or activated moderately, they are not inhibited by the addition of anti-MHC class I antibody, therefore, they are able to mediate lysis of the high MHC expressing tumors in the presence of anti-MHC class I mAb. Thus, the lack of inhibition of NK cell cytotoxic function in addition to increased expression of MHC class I on the target cells are two factors which could contribute to the observed increases in the lysis of the differentiated tumors in the presence of anti-MHC class I antibody. Lysis of differentiated tumors in the presence of anti-MHC antibodies could be due to either blocking of inhibitory MHC class I signals which activates NK cells or it could be due to the cross-linking of MHC class I on the tumor cells which delivers a death signal to the tumors or both. These possibilities are under investigation in our laboratory and are the subject of a follow up study. The observations with the anti-MHC class I antibody have significant implications regarding the function of NK and CTLs. It indicates that these cells may have a common mode of action when they encounter stem cells or undifferentiated cells, however, their mode of action may differ when they encounter differentiated cells.

In agreement with the results obtained in this report, we have previously demonstrated that the stage of maturation and differentiation of healthy untransformed stem cells as well as transformed tumorigenic cancer stem cells is predictive of their sensitivity to NK cell lysis [13, 14, 29-33]. Our work collectively suggests that anergized NK cells are as important as the non-anergized NK cells in their effector functions. NK cells are not only important for the removal and shaping of the size of the stem cells but also their differentiation. Indeed, we have previously coined the concept of split anergy in NK cells which is induced by sensitive tumor cells and after triggering of the CD16 receptor on NK cells [12, 34-37]. Induction of split anergy in NK cell effector function has significant physiological consequences since it can ultimately aid in driving differentiation of a sub-population of surviving healthy as well as transformed stem cells after initial selection by the NK cells.

Much higher amounts of NK supernatants containing IFN- $\gamma$ were required to induce resistance to NK cell mediated cytotoxicity and growth arrest in XO2GB when compared to OSCSCs. Unlike OSCSCs, higher amounts of NK supernatants containing IFN- $\gamma$ were unable to either promote detachment or induce substantial cell death in XO2GB. These experiments reveal crucial differences between tumor types in their responses to NK cell mediated differentiation, and may uncover the underlying mechanisms governing GBMs aggressive behavior and poor prognosis in patients.

The inability of patient's NK cells to contain GBM stem cells due to the proliferating cancer stem cells and conversion of NK cells to cytokine secreting cells may likely be one mechanism by which tumor cells may remain viable. Poorly differentiated tumors have unfavorable prognosis since the microenvironment of these tumors are likely to condition a great majority if not all of the NK cells to support differentiation of newly generated cancer stem cells, and as such these patients are likely to have more NK cells with no or low cytotoxic function. Therefore, these patients may benefit from repeated NK cell transplantation for elimination of cancer stem cells. In this regard depletion of NK anergizing effectors such as monocytes via radiation or chemotherapeutic drugs before NK cell transplantation should in theory provide such strategy. However, this strategy may also halt or decrease the ability of NK cells to drive differentiation of the tumors due to the removal of synergizing effect of monocytes with NK cells for secretion of TNF- $\alpha$ and IFN- $\gamma$, leading to growth of cancer stem cells [13]. Alternatively, a strong tumor differen- 
tiating microenvironment may be induced by the synergistic effect of monocytes and NK cells. The benefit of such approach is the ability of chemotherapeutic drugs to target differentiated tumors in addition to the lack of differentiated tumors to metastasize. Indeed, our recent in vivo data indicated that pancreatic cancer stem cells have the ability to grow faster and metastasize, whereas their differentiated tumors grew slower and remained localized for a long period of time without metastasizing (manuscript in prep).

It is possible that the successful cancer therapy may lie between a balance in two abovementioned approaches. The most dangerous and devastating outcome of the cancer is its ability to deplete NK cells and other synergizing immune effectors. In this case, cancer stem cells will survive and remain poorly differentiated, which are likely reasons for cancer invasion and metastasis. NK cell immunotherapy in these patients should be highly beneficial in containing tumor progression and metastasis.

\section{Supplementary Material}

Figure S1. http://www.jcancer.org/v06p0866s1.pdf

\section{Competing Interests}

The authors have declared that no competing interest exists.

\section{References}

1. Tseng HC, Bui V, Man YG, Cacalano N, Jewett A. Induction of Split Anergy Conditions Natural Killer Cells to Promote Differentiation of Stem Cells through Cell-Cell Contact and Secreted Factors. Front Immunol. 2014; 5: 269.

2. Poli A, Kmiecik J, Domingues O, Hentges F, Blery M, Chekenya M, et al. NK cells in central nervous system disorders. J Immunol. 2013; 190: 5355-62.

3. Poli A, Wang J, Domingues O, Planaguma J, Yan T, Rygh CB, et al. Targeting glioblastoma with NK cells and mAb against NG2/CSPG4 prolongs animal survival. Oncotarget. 2013; 4: 1527-46.

4. Tanaka H, Nakao M, Shichijo S, Itoh K. Nonsteroidal anti-inflammatory drugs differentially regulate cytokine production in human lymphocytes: up-regulation of TNF, IFN-gamma and IL-2, in contrast to down-regulation of IL-6 production. Cytokine. 1995; 7: 372-9.

5. Miescher S, Stoeck M, Qiao L, Barras C, Barrelet L, von Fliedner V. Preferential clonogenic deficit of CD8-positive T-lymphocytes infiltrating human solid tumors. Cancer research. 1988; 48: 6992-8.

6. Qin J, Han B, Pang J. [The relationship between TIL from human primary hepatic carcinoma and prognosis]. Zhonghua Yi Xue Za Zhi. 1997: 77: 167-70.

7. Han X, Papadopoulos AJ, Ruparelia V, Devaja O, Raju KS. Tumor lymphocytes in patients with advanced ovarian cancer: changes during in vitro culture and implications for immunotherapy. Gynecol Oncol. 1997; 65: 391-8.

8. Aggarwal S, Pittenger MF. Human mesenchymal stem cells modulate allogeneic immune cell responses. Blood. 2005; 105: 1815-22.

9. Selmani Z, Naji A, Zidi I, Favier B, Gaiffe E, Obert L, et al. Human leukocyte antigen-G5 secretion by human mesenchymal stem cells is required to suppress $\mathrm{T}$ lymphocyte and natural killer function and to induce CD4+CD25highFOXP3+ regulatory T cells. Stem Cells. 2008; 26: 212-22.

10. Spaggiari GM, Capobianco A, Abdelrazik H, Becchetti F, Mingari MC, Moretta L. Mesenchymal stem cells inhibit natural killer-cell proliferation, cytotoxicity, and cytokine production: role of indoleamine 2,3-dioxygenase and prostaglandin E2. Blood. 2008; 111: 1327-33.

11. Jewett A, Bonavida B. Target-induced inactivation and cell death by apoptosis in a subset of human NK cells. J Immunol. 1996; 156: 907-15.

12. Jewett A, Bonavida B. Target-induced anergy of natural killer cytotoxic function is restricted to the NK-target conjugate subset. Cellular immunology. 1995; 160: 91-7.

13. Tseng HC, Arasteh A, Paranjpe A, Teruel A, Yang W, Behel A, et al. Increased lysis of stem cells but not their differentiated cells by natural killer cells; de-differentiation or reprogramming activates NK cells. PLoS One. 2010; 5: e11590.

14. Jewett A, Arasteh A, Tseng HC, Behel A, Arasteh H, Yang W, et al. Strategies to rescue mesenchymal stem cells (MSCs) and dental pulp stem cells (DPSCs) from NK cell mediated cytotoxicity. PLoS One. 2010; 5: e9874.

15. Jewett A, Cacalano NA, Head C, Teruel A. Coengagement of CD16 and CD94 receptors mediates secretion of chemokines and induces apoptotic death of naive natural killer cells. Clin Cancer Res. 2006; 12: 1994-2003.

16. Jewett A, Gan XH, Lebow LT, Bonavida B. Differential secretion of TNF-alpha and IFN-gamma by human peripheral blood-derived NK subsets and association with functional maturation. J Clin Immunol. 1996; 16: 46-54.

17. Jewett A, Cavalcanti M, Bonavida B. Pivotal role of endogenous TNF-alpha in the induction of functional inactivation and apoptosis in NK cells. J Immunol. 1997; 159: 4815-22.

18. Jewett A, Bonavida B. Target-induced anergy of natural killer cytotoxic function is restricted to the NK-target conjugate subset. Cell Immunol. 1995; 160: 91-7.

19. Jewett A, Bonavida B. MHC-Class I antigens regulate both the function and the survival of human peripheral blood NK cells: role of endogenously secreted TNF-alpha. Clin Immunol. 2000; 96: 19-28.

20. Jewett A, Teruel A, Romero M, Head C, Cacalano N. Rapid and potent induction of cell death and loss of NK cell cytotoxicity against oral tumors by $\mathrm{F}\left(\mathrm{ab}^{\prime}\right) 2$ fragment of anti-CD16 antibody. Cancer immunology, immunotherapy : CII. 2008; 57: 1053-66.

21. Lai P, Rabinowich H, Crowley-Nowick PA, Bell MC, Mantovani G, Whiteside TL. Alterations in expression and function of signal-transducing proteins in tumor-associated $\mathrm{T}$ and natural killer cells in patients with ovarian carcinoma. Clin Cancer Res. 1996; 2: 161-73.

22. Kuss I, Saito T, Johnson JT, Whiteside TL. Clinical significance of decreased zeta chain expression in peripheral blood lymphocytes of patients with head and neck cancer. Clin Cancer Res. 1999; 5: 329-34.

23. Oka N, Soeda A, Inagaki A, Onodera M, Maruyama H, Hara A, et al. VEGF promotes tumorigenesis and angiogenesis of human glioblastoma stem cells. Biochem Biophys Res Commun. 2007; 360: 553-9.

24. Jewett A, Bonavida B. Interferon-alpha activates cytotoxic function but inhibits interleukin-2-mediated proliferation and tumor necrosis factor-alpha secretion by immature human natural killer cells. J Clin Immunol. 1995; 15: 35-44.

25. Jewett A, Wang MY, Teruel A, Poupak Z, Bostanian Z, Park NH. Cytokine dependent inverse regulation of CD54 (ICAM1) and major histocompatibility complex class I antigens by nuclear factor kappaB in HEp2 tumor cell line: effect on the function of natural killer cells. Hum Immunol. 2003; 64: 505-20.

26. Soeda A, Inagaki A, Oka N, Ikegame Y, Aoki H, Yoshimura S, et al. Epidermal growth factor plays a crucial role in mitogenic regulation of human brain tumor stem cells. J Biol Chem. 2008; 283: 10958-66.

27. Inagaki A, Soeda A, Oka N, Kitajima H, Nakagawa J, Motohashi T, et al. Long-term maintenance of brain tumor stem cell properties under at non-adherent and adherent culture conditions. Biochem Biophys Res Commun. 2007; 361: 586-92.

28. Avril T, Vauleon E, Hamlat A, Saikali S, Etcheverry A, Delmas C, et al. Human glioblastoma stem-like cells are more sensitive to allogeneic NK and T cell-mediated killing compared with serum-cultured glioblastoma cells. Brain pathology. 2012; 22: 159-74.

29. Jewett A, Tseng H.C. Tumor Microenvironment may Shape the Function and Phenotype of NK Cells Through the Induction of Split Anergy and Generation of Regulatory NK Cells. In: Shurin M, Viktor Umansky and Anatoli Malyguine, editor. The Tumor Immunoenvironment: Springer; 2013: 361-84.

30. Jewett A, Man YG, Tseng HC. Dual functions of natural killer cells in selection and differentiation of stem cells; role in regulation of inflammation and regeneration of tissues. Journal of Cancer. 2013; 4: 12-24.

31. Jewett A, Tseng HC. Potential rescue, survival and differentiation of cancer stem cells and primary non-transformed stem cells by monocyte-induced split anergy in natural killer cells. Cancer Immunol Immunother. 2012; 61: 265-74.

32. Jewett A, Tseng HC, Arasteh A, Saadat S, Christensen RE, Cacalano NA. Natural killer cells preferentially target cancer stem cells; role of monocytes in protection against NK cell mediated lysis of cancer stem cells. Current drug delivery. 2012; 9: 5-16

33. Jewett A, Man YG, Cacalano N, Kos J, Tseng HC. Natural killer cells as effectors of selection and differentiation of stem cells: Role in resolution of inflammation. Journal of immunotoxicology. 2014.

34. Jewett A, Bonavida B. Target-induced inactivation and cell death by apoptosis in a subset of human NK cells. J Immunol. 1996; 156: 907-15.

35. Jewett A, Teruel A, Romero M, Head C, Cacalano N. Rapid and potent induction of cell death and loss of NK cell cytotoxicity against oral tumors by $\mathrm{F}\left(\mathrm{ab}^{\prime}\right) 2$ fragment of anti-CD16 antibody. Cancer Immunol Immunother. 2008; 57: 1053-66.

36. Jewett A, Cavalcanti M, Bonavida B. Pivotal role of endogenous TNF-alpha in the induction of functional inactivation and apoptosis in NK cells. J Immunol. 1997; 159: 4815-22.

37. Jewett A, Cacalano NA, Head C, Teruel A. Coengagement of CD16 and CD94 receptors mediates secretion of chemokines and induces apoptotic death of naive natural killer cells. Clinical cancer research : an official journal of the American Association for Cancer Research. 2006; 12: 1994-2003. 